\title{
PREVALÊNCIA E FATORES ASSOCIADOS À DISCRIMINAÇÃO RACIAL PERCEBIDA NOS SERVIÇOS DE SAÚDE DO BRASIL
}

\section{Prevalence and factors associated with racial discrimination perceived in Brazil's health services}

\section{Prevalencia y factores asociados con la discriminación racial percibida en los servicios de salud de Brasil}

\author{
Marianny Nayara Paiva Dantas (iD \\ Universidade Federal do Rio Grande do Norte - UFRN - Natal (RN) - Brasil
}

Kezauyn Miranda Aiquoc iD

Universidade Federal do Rio Grande do Norte - UFRN - Natal (RN) - Brasil

Emelynne Gabrielly de Oliveira Santos (iD

Universidade Federal do Rio Grande do Norte - UFRN - Natal (RN) - Brasil

\section{Mercês de Fátima dos Santos Silva (iD}

Universidade Federal do Rio Grande do Norte - UFRN - Natal (RN) - Brasil

Faculdade de Ciências da Saúde do Trairi - FACISA - Santa Cruz (RN) - Brasil

Dyego Leandro Bezerra de Souza (iD

Universidade Federal do Rio Grande do Norte - UFRN - Natal (RN) - Brasil

Nayre Beatriz Martiniano de Medeiros (iD

Centro Universitário do Rio Grande do Norte - UNIRN - Natal (RN) - Brasil

Isabelle Ribeiro Barbosa iD

Universidade Federal do Rio Grande do Norte - UFRN - Natal (RN) - Brasil

\section{RESUMO}

Objetivo: Analisar a prevalência da discriminação por raça/cor praticada por prestadores de cuidados em serviços de saúde no Brasil e seus fatores associados. Métodos: Estudo transversal realizado a partir dos dados da Pesquisa Nacional de Saúde (PNS) de 2013, com variável dependente elaborada a partir da resposta à questão X25: $O(A) \operatorname{Sr}(a)$ já se sentiu discriminado (a) ou tratado(a) pior do que as outras pessoas no serviço de saúde? As variáveis independentes foram: região de moradia no país, situação de domicílio, raça/cor, sexo, faixa etária, vive com cônjuge, ocupação, escolaridade, critério de classe social no Brasil, tabagismo, alcoolismo, presença de multimorbidade, autoavaliação de saúde e uso de plano privado de saúde médico ou odontológico. Esses fatores foram analisados através das razões de prevalência (RP), com análise multivariada pela regressão de Poisson (IC95\%), com teste de Wald para estimação robusta. Resultados: A prevalência da discriminação por raça/cor foi de $1,45 \%$ ( $n=6055$ ), associada a ser negro (RP 3,74 IC95\% 2,89-4,85), ter idade entre 25-39 anos (RP 1,89 IC95\% 1,21-2,55), ser fumante (RP 1,55 IC95\% 1,17-2,09), possuir quatro morbidades (RP 2,54 IC95\% 1,62-3,99), avaliar a própria saúde como ruim ou muito ruim (RP 1,76 IC95\% 1,25-2,48), ser usuário do serviço público de saúde (RP 1,33 IC95\% 1,02-1,73) e residir na zona urbana do país (RP 1,48 IC95\% 1,10-1,98). Conclusão: A discriminação por raça/cor praticada por prestadores de cuidados de saúde no Brasil apresenta baixa prevalência, o que pode estar relacionado às construções e sanções culturais, sociais e legais envolvidas nesse fenômeno.

Descritores: Racismo; Serviços de Saúde; Fatores Socioeconômicos; Preconceito; Brasil.

\section{ABSTRACT}

Objective: To analyze the prevalence of race/color discrimination practiced by healthcare providers in Brazil and its associated factors. Methods: Cross-sectional study based on data from the 2013 National Health Survey (PNS). The dependent variable was elaborated from the answer to question X25 - Have you ever felt discriminated against or treated worse than other people in

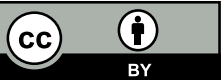


the health service? The independent variables used in the study were: region of residence in the country, domicile situation, skin color, sex, age group, living with spouse, occupation, education, social class criterion in Brazil, smoking, alcoholism, presence of multimorbidity, self-rated health and use of a private medical or dental health plan. These factors were analyzed through the Prevalence Ratios (PR), with Poisson Regression multivariate analysis (95\% Cl), using the Wald test for robust estimation. Results: The prevalence of race/color discrimination was $1.45 \% \%(n=6055)$, associated with being black (PR 3.74 95\% Cl 2.89-4.85), aged 25-39 years (PR 1.89 95\% Cl 1.21-2.55), being a smoker (PR 1.55 95\% Cl 1.17-2.09), having four morbidities (PR $2.5495 \%$ Cl 1.62-3 99), evaluate their health as poor or very poor (PR $1.7695 \% \mathrm{Cl} 1.25-2.48$ ), be a public health service user (PR $1.3395 \%$ $\mathrm{Cl}$ 1.02-1.73), and reside in the urban area of the country (PR 1.48 95\% Cl 1.10-1.98). Conclusion: It was possible to identify that the discrimination by race/color practiced by healthcare providers in Brazil has low prevalence, which may be related to the cultural, social and legal constructions and sanctions involved in this phenomenon.

Descriptors: Racism; Health Services; Socioeconomic Factors; Prejudice; Brazil.

\section{RESUMEN}

Objetivo: Analizar la prevalencia de la discriminación de raza/color practicada por los prestadores de cuidados de servicios de salud de Brasil y sus factores asociados. Métodos: Estudio transversal realizado a partir de los datos de la Investigación Nacional de Salud (INS) de 2013 con la variable dependiente elaborada a partir de la respuesta de la pregunta X25: Usted ya se sintió discriminado(a) o tratado(a) peor que las otras personas en el servicio de salud? Las variables independientes fueron: la región de vivienda en el país, la situación del hogar, la raza/color, el sexo, la franja de edad, si vive con pareja, la ocupación, la escolaridad, el criterio de clase social en Brasil, el tabaquismo, el alcoholismo, la presencia de multimorbidad, la auto evaluación de la salud y el uso de seguro privado de salud médico o de salud bucal. Eses factores han sido analizados a través de las razones prevalencia (RP), el análisis multivariado por la regresión de Poisson (IC95\%), la prueba de Wald para estimación robusta. Resultados: La prevalencia de la discriminación de raza/color ha sido del 1,45\% $(n=6055)$ asociada con el hecho de tener el color negro (RP 3,74 IC95\% 2,89-4,85), tener la edad entre 25-39 años (RP 1,89 IC95\% 1,21-2,55), ser fumador (RP 1,55 IC95\% 1,17-2,09), tener cuatro morbidades (RP 2, 54 IC95\% 1,62-3,99), evaluar la propia salud como mala o muy mala (RP 1,76 IC95\% 1,25-2,48), ser usuario del servicio público de salud (RP 1,33 IC95\% 1,02-1,73) y vivir en la zona urbana del país (RP 1,48 IC95\% 1,10-1,98). Conclusión: La discriminación de raza/color practicada por los prestadores de cuidados de salud de Brasil presenta baja prevalencia lo que puede estar relacionado con las construcciones y sanciones culturales, sociales $y$ legales de ese fenómeno.

Descriptores: Racismo; Servicios de Salud; Factores Socioeconómicos; Prejuicio; Brasil.

\section{INTRODUÇÃO}

O racismo apresenta-se como uma ideologia de inferioridade social utilizada para justificar o tratamento depreciativo concedido a membros de grupos raciais e étnicos, e que contribui para o agravamento e manutenção de desvantagens de poder, recursos ou oportunidades entre esses grupos ${ }^{(1,2)}$.

Esse fenômeno pode se materializar em três dimensões: através de preconceitos internalizados, estereótipos e sentimentos negativos vinculados a características raciais ou étnicas de um grupo; pela manifestação interpessoal de comportamentos e práticas discriminatórias, que excluem e inferiorizam esses grupos, concedendo-os atributos de menor valor ou considerando-os sem valor em detrimento das características de outros; e, como componente estrutural, legitimado e praticado por organizações, políticas e normas, por meio de tratamento não equitativo, discriminatório, negligente, com desvantagem no acesso a benefícios e morosidade na implementação de ações e políticas que favorecem às suas vítimas ${ }^{(1-4)}$.

De acordo com o último censo populacional realizado no Brasil, mais de $50 \%$ do contingente populacional do país é composto por indivíduos pretos e pardos. Entretanto esse grupo populacional apresenta piores indicadores de saúde(5,6).

No âmbito da saúde pública, a discriminação de raça/cor praticada por prestadores de cuidado de saúde vem sendo internacionalmente estudada, por ser considerada geradora e/ou potencializadora de agravos à saúde, da precarização das autoavaliações de saúde, de prejuízos na qualidade da prestação de serviços em saúde e do nível de satisfação e confiança nos serviços de saúde por grupos étnicos ${ }^{(7-12)}$.

Ademais, a assistência à saúde equitativa e universal são prerrogativas anunciadas por marcos da criação e regulação do Sistema Único de Saúde (SUS) do Brasil. Mesmo com a existência desse arcabouço legal robusto, são identificadas disparidades em saúde entre grupos raciais, o que indica a não efetivação dos princípios defendidos pela Constituição Federal do Brasil(13). 
Todavia as disparidades nas condições de vida e saúde da população negra brasileira são reconhecidamente influenciadas pelo racismo estrutural(13). Na sociedade brasileira essa estruturação ocorreu por todo o período escravagista, perpassando a abolição, pois a segregação da população em duas raças (brancos e negros) conseguiu se manter mesmo em uma época em que o racismo se tornou crime inafiançável(14).

O crime de discriminação racial ainda tem sido frequente o Brasil. O Ministério Público do Trabalho de Mato Grosso do Sul divulgou um balanço dos últimos cinco anos e, nesse período, foram recebidas 896 denúncias por discriminação em razão da origem, raça, cor ou etnia. No ano de 2014, ocorreram 157 denúncias, e esse número cresceu cerca de $30,5 \%$ até o ano de 2018 , alcançando a marca de 205 denúncias ${ }^{(15)}$.

Para ratificar o compromisso do país em seu enfrentamento, a Política Nacional de Saúde Integral da População Negra (PNSIPN) apresenta-se como uma estratégia no combate às desigualdades no SUS, com vistas à melhoria do acesso e da condição saúde dessa população, pois compreende que a assistência integral, livre de discriminação racial, é indispensável para a redução das disparidades de saúde decorrentes da raça/cor ${ }^{(16)}$.

Considerando a complexidade das relações raciais existentes no Brasil, a importância do quesito "cor/raça" na elaboração do racismo e na prática da discriminação no país, torna-se relevante reconhecer essa iniquidade social, identificar as características associadas a esse fenômeno, permitindo reflexões sobre seus efeitos, bem como a elaboração de estratégias de intervenção, com gestores e prestadores de cuidados de saúde, para seu enfrentamento. Dessa forma, o objetivo do presente estudo foi analisar a prevalência da discriminação por raça/cor praticada por prestadores de cuidados em serviços de saúde do Brasil e seus fatores associados.

\section{MÉTODOS}

Trata-se de um estudo transversal que utilizou os dados da Pesquisa Nacional de Saúde (PNS) do Brasil, desenvolvida pelo Instituto Brasileiro de Geografia e Estatística (IBGE) com o Ministério da Saúde em 2013. A pesquisa é um inquérito de base domiciliar, de âmbito nacional, que caracterizou a situação de saúde, estilos de vida, vigilância de doenças crônicas, fatores de risco e acesso e uso dos serviços de saúde da população brasileira, viabilizando meios para conhecer o perfil da população nesses quesitos ${ }^{(17)}$. A análise dos dados utilizados na presente pesquisa foi realizada em outubro de 2018.

A população pesquisada na PNS compreendeu moradores de domicílios particulares do país e em idade adulta ( $\geq 18$ anos de idade), excluindo os localizados nos setores censitários especiais (quartéis, bases militares, alojamentos, acampamentos, embarcações, penitenciárias, colônias penais, presídios, cadeias, asilos, orfanatos, conventos e hospitais). Realizou-se amostragem conglomerada em três estágios, sendo atribuídos pesos a cada unidade amostral. No primeiro estágio, selecionaram-se os setores censitários (unidade territorial de coleta das operações censitárias, definido pelo IBGE), formando as unidades primárias de amostragem (UPAs); no segundo, selecionaram-se os domicílios e, no terceiro, os moradores adultos. Em todas as etapas, fez-se a seleção das subamostras por amostragem aleatória simples (AAS) ${ }^{(17)}$.

O tamanho definido para a amostra foi de 81.357 domicílios, considerando um indivíduo por domicílio. Ao final da coleta, realizou-se entrevista em 60.202 domicílios, decorrendo em uma taxa de resposta de $86 \%(6,17)$.

Dividiu-se o questionário da PNS em módulos, os quais contemplam características do domicílio, de todos os moradores e do morador adulto selecionado no estudo, totalizando 743 questões. Os entrevistadores foram treinados para a realização da entrevista e para o uso de computadores de mão, nos quais eram inseridos os dados coletados ${ }^{(18)}$.

O presente estudo analisou questões da PNS referentes à discriminação em serviços de saúde, presente no "Módulo X" do questionário, que contém 24 perguntas sobre atendimento médico, acesso ao atendimento e avaliação sobre $o$ atendimento recebido no serviço de saúde ${ }^{(18)}$.

Para a construção da variável dependente denominada "discriminação por raça/cor praticada por prestadores de cuidados de saúde no Brasil", utilizou-se a seguinte pergunta do questionário: "X25 - O(A) Sr(a) já se sentiu discriminado (a) ou tratado(a) pior do que as outras pessoas no serviço de saúde, por algum médico ou outro profissional de saúde, por algum destes motivos?". A pergunta contemplava como alternativas as respostas "sim" ou "não" para dez motivos de discriminação, ordenados de "a" a "j", são eles: a) falta de dinheiro, b) classe social, c) raça/cor, d) tipo de ocupação, e) tipo de doença, f) preferência sexual, g) religião/crença, h) sexo, i) idade e j) outros ${ }^{(19)}$. Para identificar a discriminação por raça/cor, o indicador foi calculado a partir das respostas "sim" ao o "item c", correspondente à discriminação por raça/cor ${ }^{(18)}$. Dos 60.202 indivíduos entrevistados na PNS, 6.055 afirmaram haver sofrido discriminação por "raça/cor". 
Utilizaram-se, neste estudo, as variáveis independentes: região de moradia no país (Centro-Oeste, Sul, Sudeste, Norte, Nordeste), situação de domicílio (zona rural ou urbana), raça/cor (negros - pretos e pardos; e não negros brancos, amarelos e indígenas), sexo (masculino ou feminino), faixa etária (18-20, 30-39, 40-59, 60 ou mais), vive com cônjuge (sim ou não), ocupação (ocupado, desocupado), escolaridade (sem escolaridade, ensino fundamental, ensino médio, ensino superior ou mais), critério de classe social no Brasil ( $\mathrm{e}$ e $B, C, D$ e E), uso de tabaco e seus derivados (fumante, ex-fumante, nunca fumou), uso de bebida alcoólica (uso excessivo, uso moderado, nunca bebeu), presença de multimorbidade (nenhuma, duas, três, quatro ou mais multimorbidades), autoavaliação de saúde (muito boa/boa, regular, ruim/muito ruim) e uso de plano privado de saúde médico ou odontológico (sim ou não).

Para análise estatística, utilizou-se a análise das prevalências da discriminação por raça/cor praticada por prestadores de cuidados de saúde em relação às características socioeconômicas e condições de saúde da população estudada. Posteriormente, realizou-se a análise bivariada para obtenção das razões de prevalência (RP) com respectivos intervalos de confiança de 95\% (IC 95\%) e valores de $p(p<0,05)$. Para as variáveis com significância estatística na análise bivariada (valor de $p<0,2$ ), realizou-se a análise multivariada por meio da regressão de Poisson, com teste de Wald para estimação robusta, uma vez que, a partir dessa análise, é possível obter uma maior sensibilidade dos dados, sendo considerados nas análises os pesos amostrais resultantes do delineamento da amostra complexa da PNS. Para análise estatística, utilizou-se o programa Stata, versão 14, através do comando complex sample.

O projeto da PNS obteve a aprovação da Comissão Nacional de Ética em Pesquisa (CONEP), no dia 8 de julho de 2013, com o n. ${ }^{\circ}$ 10853812.7.0000.0008.

O presente estudo utiliza dados secundários da PNS disponíveis em sites oficiais do Ministério da Saúde (MS) do Brasil, portanto, sendo dispensado de apreciação em comitê de ética em pesquisa, em conformidade com a Resolução n. ${ }^{\circ} 466 / 2012$ do Conselho Nacional de Saúde.

\section{RESULTADOS}

A Tabela I apresenta a prevalência geral da discriminação por cor/raça praticada por prestadores de cuidados de saúde no Brasil, conforme as características socioeconômicas e de saúde da população. Identificou-se esse tipo de discriminação em 1,45\% ( $n=6055$; IC95\% 1,29-1,62) na população estudada, com maior propensão a serem discriminados os moradores de zona urbana $(1,48 \%)$, com maior prevalência no Norte do país $(2,12 \%)$, seguidos pela região CentroOeste $(1,92 \%)$ e Nordeste $(1,6 \%)$.

A análise descritiva confirma que relataram em maior proporção haver sofrido discriminação por raça/cor praticada por prestadores de cuidados de saúde: pessoas negras (pretas e pardas) (2,29\%; IC95\% 2,02-2,59), ter entre 25 -39 anos (1,82\%; IC95\% 1,56-2,12), sem escolaridade (1,69\%; IC95\%1,24-2,31), fumantes (2,29\%; IC95\% $1,81-2,90)$, que bebem excessivamente (1,78\%; IC95\%1,26-2,50), que avaliam sua saúde como ruim/muito ruim (2,80\%; IC95\%2,17-3,62), que possuem quatro morbidades (2,64\%; IC95\%1,76-3,94) e que não possuem plano de saúde privado médico ou odontológico (1,65\%; IC95\%1,46-1,88). (Tabela I).

A Tabela II apresenta os resultados da análise bivariada da relação entre discriminação por raça/cor praticada por prestadores de cuidados de saúde no Brasil e fatores socioeconômicos e de saúde.

Observa-se associação estatisticamente significativa entre maior discriminação por raça/cor e residir na região Norte (RP 1,66; IC95\% 1,17-1,35) e Centro-Oeste (RP 1,50; IC95\% 1,07-2,11), ter cor da pele negra (RP 4,06; IC95\% 3,15-5,22), ter entre 25-39 anos (RP 2,17; IC95\% 1,56-3,01), ser fumante (RP 1,80; IC95\% 1,37-2,36), não possuir plano privado de saúde médico ou odontológico (RP 1,72; IC95\% 1,33-2,22), possuir quatro morbidades (RP 2,17; IC95\% 1,56-3,01) e autoavaliar a saúde como ruim/muito ruim (RP 2,45; IC95\% 1,54-3,28).

As variáveis que se apresentaram estatisticamente significativas (valor de $p<0,2$ ) na análise bivariada passaram por uma análise multivariada, e os resultados estão representados na Tabela III.

Nessa última análise, os fatores que apresentaram associação com o desfecho estudado foram: ser negro (RP 3,74; IC95\% 2,89-4,85), ter entre 25-39 anos (RP 1,89; IC95\% 1,21-2,55), fumante (RP 1,55; IC95\% 1,17-2,09), que possui quatro morbidades (RP 2,54; IC 95\% 1,62-3,99), que autoavalia sua saúde como ruim/muito ruim (RP 1,76; IC95\% 1,25-2,48), que é usuário exclusivo do serviço público de saúde (RP 1,33; IC95\% 1,02-1,73) e que reside nas zonas urbanas do país (RP 1,48; IC95\% 1,10-1,98). 
Tabela I - Prevalência (\%) da discriminação por raça/cor praticada por prestadores de cuidados de saúde, conforme as variáveis independentes da Pesquisa Nacional de Saúde, 2013.

\begin{tabular}{|c|c|c|c|}
\hline \multirow{2}{*}{ Variáveis } & \multirow{2}{*}{$P(\%)$} & \multicolumn{2}{|c|}{ IC (95\%) } \\
\hline & & LI & LS \\
\hline Discriminação racial & 1,45 & 1,29 & 1,62 \\
\hline \multicolumn{4}{|l|}{ Região } \\
\hline Centro-Oeste & 1,92 & 1,46 & 2,51 \\
\hline Sul & 1,09 & 0,79 & 1,50 \\
\hline Sudeste & 1,28 & 1,04 & 1,56 \\
\hline Norte & 2,12 & 1,60 & 2,81 \\
\hline Nordeste & 1,60 & 1,31 & 1,95 \\
\hline \multicolumn{4}{|l|}{ Situação de domicílio } \\
\hline Urbana & 1,48 & 1,31 & 1,67 \\
\hline Rural & 1,22 & 0,95 & 1,57 \\
\hline \multicolumn{4}{|l|}{ Cor/raça } \\
\hline Negros & 2,29 & 2,02 & 2,59 \\
\hline Não negros & 0,56 & 0,45 & 0,70 \\
\hline \multicolumn{4}{|l|}{ Sexo } \\
\hline Feminino & 1,55 & 1,35 & 1,78 \\
\hline Masculino & 1,32 & 1,13 & 1,55 \\
\hline \multicolumn{4}{|l|}{ Idade } \\
\hline $18-24$ & 0,95 & 0,70 & 1,29 \\
\hline $25-39$ & 1,82 & 1,56 & 2,12 \\
\hline $40-59$ & 1,65 & 1,38 & 1,97 \\
\hline 60 ou + & 0,84 & 0,62 & 1,14 \\
\hline \multicolumn{4}{|l|}{ Vive com cônjuge } \\
\hline Não & 1,40 & 1,19 & 1,64 \\
\hline Sim & 1,47 & 1,27 & 1,71 \\
\hline \multicolumn{4}{|l|}{ Ocupação } \\
\hline Ocupado & 1,43 & 1,26 & 1,63 \\
\hline Desocupado & 1,47 & 1,23 & 1,75 \\
\hline \multicolumn{4}{|l|}{ Escolaridade } \\
\hline Sem escolaridade & 1,69 & 1,24 & 2,31 \\
\hline Ensino fundamental & 1,58 & 1,34 & 1,86 \\
\hline Ensino médio & 1,49 & 1,24 & 1,77 \\
\hline Ensino superior ou mais & 1 & 0,79 & 1,28 \\
\hline \multicolumn{4}{|l|}{ Critério classe econômica } \\
\hline$A \in B$ & 1,33 & 1,06 & 1,67 \\
\hline C & 1,44 & 1,20 & 1,73 \\
\hline$D$ e $E$ & 1,53 & 1,30 & 1,79 \\
\hline \multicolumn{4}{|l|}{ Tabagismo } \\
\hline Nunca fumou & 1,28 & 1,11 & 1,46 \\
\hline Fumante & 2,29 & 1,81 & 2,90 \\
\hline Ex-fumante & 1,39 & 1,08 & 1,79 \\
\hline \multicolumn{4}{|l|}{ Uso de álcool } \\
\hline Não bebe & 1,48 & 1,30 & 1,69 \\
\hline Moderado & 1,15 & 0,86 & 1,56 \\
\hline Excessivo & 1,78 & 1,26 & 2,50 \\
\hline \multicolumn{4}{|l|}{ Multimorbidades } \\
\hline Nenhuma morbidade & 1,21 & 1,07 & 1,37 \\
\hline Duas morbidades & 2,25 & 1,74 & 2,91 \\
\hline
\end{tabular}




\begin{tabular}{|c|c|c|c|}
\hline Três morbidades & 1,69 & 1,19 & 2,41 \\
\hline Quatro morbidades & 2,64 & 1,76 & 3,94 \\
\hline \multicolumn{4}{|c|}{ Autoavaliação de saúde } \\
\hline Muito boa & 1,14 & 0,99 & 1,32 \\
\hline Regular & 1,88 & 1,56 & 2,25 \\
\hline Ruim/muito ruim & 2,80 & 2,17 & 3,62 \\
\hline \multicolumn{4}{|c|}{ Plano de saúde privado, médico ou odontológico } \\
\hline Sim & 0,96 & 0,77 & 1,20 \\
\hline Não & 1,65 & 1,46 & 1,88 \\
\hline
\end{tabular}

P: Prevalência; IC: intervalo de Confiança; LS: limite superior; LI: limite inferior

Tabela II - Análise bivariada dos fatores relacionados à discriminação por raça/cor praticada por prestadores de cuidados de saúde, conforme as variáveis independentes da Pesquisa Nacional de Saúde, 2013.

\begin{tabular}{|c|c|c|c|c|}
\hline \multirow{2}{*}{ Variáveis } & \multirow{2}{*}{ RP } & \multicolumn{2}{|c|}{ IC (95\%) } & \multirow{2}{*}{ p-valor } \\
\hline & & LI & LS & \\
\hline \multicolumn{5}{|l|}{ Região } \\
\hline Sudeste & 1 & & & \multirow{5}{*}{$<0,001$} \\
\hline Centro-Oeste & 1,5 & 1,07 & 2,11 & \\
\hline Sul & 0,85 & 0,59 & 1,25 & \\
\hline Norte & 1,66 & 1,17 & 2,35 & \\
\hline Nordeste & 1,25 & 0,95 & 1,66 & \\
\hline \multicolumn{5}{|l|}{ Situação de domicílio } \\
\hline Rural & 1 & & & \multirow[t]{2}{*}{0,178} \\
\hline Urbana & 1,21 & 0,92 & 1,6 & \\
\hline \multicolumn{5}{|l|}{ Raça/cor } \\
\hline Não negros & 1 & & & \multirow[t]{2}{*}{$<0,001$} \\
\hline Negros & 4,06 & 3,15 & 5,22 & \\
\hline \multicolumn{5}{|l|}{ Sexo } \\
\hline Masculino & 1 & & & \multirow[t]{2}{*}{0,110} \\
\hline Feminino & 1,17 & 0,97 & 1,42 & \\
\hline \multicolumn{5}{|l|}{ Idade } \\
\hline $60+$ & 1 & & & \multirow{4}{*}{$<0,001$} \\
\hline $18-24$ & 1,14 & 0,74 & 1,74 & \\
\hline $25-39$ & 2,17 & 1,56 & 3,01 & \\
\hline $40-59$ & 1,97 & 1,39 & 2,79 & \\
\hline \multicolumn{5}{|l|}{ Vive com cônjuge } \\
\hline Não & 1 & & & \multirow[t]{2}{*}{0,610} \\
\hline Sim & 1,06 & 0,86 & 1,3 & \\
\hline \multicolumn{5}{|l|}{ Ocupação } \\
\hline Ocupado & 1 & & & \multirow[t]{2}{*}{0,820} \\
\hline Desocupado & 1,02 & 0,83 & 1,26 & \\
\hline \multicolumn{5}{|l|}{ Educação } \\
\hline Sem escolaridade & 1 & & & \multirow{4}{*}{0,010} \\
\hline Ensino fundamental & 0,93 & 0,66 & 1,31 & \\
\hline Ensino médio & 0,88 & 0,63 & 1,22 & \\
\hline Ensino superior & 0,59 & 0,4 & 0,88 & \\
\hline \multicolumn{5}{|l|}{ Critério Brasil } \\
\hline A e B & 1 & & & \multirow{3}{*}{0,610} \\
\hline C & 1,08 & 0,81 & 1,44 & \\
\hline De E & 1,15 & 0,87 & 1,51 & \\
\hline \multicolumn{5}{|l|}{ Tabagismo } \\
\hline Nunca fumou & 1 & & & \multirow{3}{*}{$<0,001$} \\
\hline Fumante & 1,80 & 1,37 & 2,36 & \\
\hline Ex-fumante & 1,09 & 0,83 & 1,44 & \\
\hline
\end{tabular}




\begin{tabular}{|c|c|c|c|c|}
\hline \multicolumn{5}{|l|}{ Uso de álcool } \\
\hline Não bebe & 1 & & & \multirow{3}{*}{0,14} \\
\hline Moderado & 0,77 & 0,56 & 1,06 & \\
\hline Excessivo & 1,20 & 0,83 & 1,73 & \\
\hline \multicolumn{5}{|l|}{ Multimorbidade } \\
\hline Nenhuma morbidade & 1 & & & \multirow{4}{*}{$<0,001$} \\
\hline Duas morbidades & 1,86 & 1,43 & 2,42 & \\
\hline Três morbidades & 1,40 & 0,97 & 2,01 & \\
\hline Quatro morbidades & 2,18 & 1,43 & 3,32 & \\
\hline \multicolumn{5}{|l|}{ Autoavaliação da saúde } \\
\hline Boa/muito boa & 1 & & & \multirow{3}{*}{$<0,001$} \\
\hline Regular & 1,64 & 1,32 & 2,04 & \\
\hline Ruim/muito ruim & 2,45 & 1,84 & 3,28 & \\
\hline \multicolumn{5}{|c|}{ Plano de Saúde privado, médico ou odontológico } \\
\hline $\operatorname{Sim}$ & 1 & & & \multirow[t]{2}{*}{$<0,001$} \\
\hline Não & 1,72 & 1,33 & 2,22 & \\
\hline
\end{tabular}

RP: Razão de prevalência; IC: intervalo de Confiança; LS: limite superior; LI: limite inferior; p valor: probabilidade de significância

Tabela III - Modelo multivariado pela Regressão de Poisson com as Razões de Prevalência ajustadas (RPaj) das variáveis associadas à discriminação por raça/cor praticada por prestadores de cuidados de saúde. Pesquisa Nacional de Saúde, 2013.

\begin{tabular}{|c|c|c|c|c|}
\hline \multirow{2}{*}{ Variáveis } & \multirow{2}{*}{ RPaj } & \multicolumn{2}{|c|}{ IC $(95 \%)$} & \multirow{2}{*}{ p-valor } \\
\hline & & LI & LS & \\
\hline \multicolumn{5}{|l|}{ Situação de domicílio } \\
\hline Rural & 1 & & & \multirow[t]{2}{*}{$<0,010$} \\
\hline Urbana & 1,48 & 1,1 & 1,98 & \\
\hline \multicolumn{5}{|l|}{ Cor/Raça } \\
\hline Não negro & 1 & & & \multirow[t]{2}{*}{$<0,001$} \\
\hline Negros & 3,74 & 2,89 & 4,85 & \\
\hline \multicolumn{5}{|l|}{ Sexo } \\
\hline Masculino & 1 & & & \multirow[t]{2}{*}{0,30} \\
\hline Feminino & 1,11 & 0,91 & 1,35 & \\
\hline \multicolumn{5}{|l|}{ Idade } \\
\hline $18-25$ & 1 & & & \multirow{4}{*}{$<0,001$} \\
\hline $25-39$ & 1,81 & 1,29 & 2,55 & \\
\hline $40-59$ & 1,31 & 0,89 & 1,94 & \\
\hline 60-maiores & 0,59 & 0,35 & 0,98 & \\
\hline \multicolumn{5}{|l|}{ Tabagismo } \\
\hline Nunca fumou & 1 & & & \multirow{3}{*}{$<0,010$} \\
\hline Fumante & 1,57 & 1,17 & 2,09 & \\
\hline Ex-fumante & 1,04 & 1,41 & 0,77 & \\
\hline \multicolumn{5}{|l|}{ Multimorbidade } \\
\hline Nenhuma morbidade & 1 & & & \multirow{4}{*}{$<0,001$} \\
\hline Duas morbidades & 1,97 & 1,49 & 2,59 & \\
\hline Três morbidades & 1,63 & 1,11 & 2,38 & \\
\hline Quatro morbidades & 2,54 & 1,62 & 3,99 & \\
\hline \multicolumn{5}{|l|}{ Autoavaliação de saúde } \\
\hline Boa/muito boa & 1 & & & \multirow{3}{*}{$<0,010$} \\
\hline Regular & 1,32 & 1,06 & 1,64 & \\
\hline Ruim/muito ruim & 1,76 & 1,25 & 2,48 & \\
\hline \multicolumn{5}{|c|}{ Plano de saúde privado, médico ou odontológico } \\
\hline Sim & 1 & & & \multirow[t]{2}{*}{0,04} \\
\hline Não & 1,33 & 1,02 & 1,73 & \\
\hline
\end{tabular}

RPaj: razão de prevalência ajustada; IC: intervalo de Confiança; LS: limite superior; LI: limite inferior; Valor de p: probabilidade de significância 


\section{DISCUSSÃO}

O estudo da prevalência e dos fatores associados à discriminação racial praticada por prestadores de cuidados de saúde no Brasil permitiu observar um padrão de baixa prevalência $(1,45 \%)$ quando comparada àquelas observadas em estudos americanos.

Um estudo ${ }^{(19)}$ aponta que a discriminação por raça/cor praticada por prestadores de cuidados de saúde apresentou uma prevalência geral de $10,38 \%$, enquanto que, para a população negra, essa prevalência foi de $25,41 \%$. Ademais, outro estudo ${ }^{(2)}$ identificou que $4,7 \%$ das pessoas foram vítimas de discriminação em serviços de saúde e que esse valor correspondeu a $9,7 \%$ entre negros.

Múltiplos acontecimentos sociais e históricos podem contribuir para minimizar tanto a prática quanto a percepção da discriminação por raça/cor entre os brasileiros quando comparado aos Estados Unidos da América (EUA). No Brasil, evidenciou-se, após o fim da escravidão, uma suposta "democracia racial", que inclui a ideia de igualdade entre seus cidadãos, independente da raça/cor, mascarando a percepção e a prática do fenômeno(5). Além disso, o processo de miscigenação, evidente na realidade brasileira, não se processou da mesma forma nos EUA, onde esteve acompanhado da intensa segregação racial, o que resultou em um profundo sentimento de identidade étnica de sua população negra e ampliou sua percepção sobre o fenômeno da discriminação racial ${ }^{(19,20)}$.

Ademais, as reflexões sobre discriminação racial já aconteciam desde final do século XIX no contexto norteamericano, permitindo a compreensão e a percepção do fenômeno por sua população; enquanto que, no Brasil, os debates sobre a discriminação vivida pela população negra são recentes e ainda escassos, tornando essa prática cada vez mais sutil, velada e, por vezes, envolta em ambiguidade, dificultando a percepção pelas vítimas e eximindo seus perpetradores de sanções sociais e penais ${ }^{(21)}$.

Nos espaços de prestação de cuidados em saúde, a discriminação racial pode materializar-se pelo comportamento profissional através de olhares e silêncio durante o atendimento, assim como pelo desconhecimento e despreparo acerca das condições de saúde peculiares à população negra, prejudicando a avaliação e a abordagem do seu processo de saúde-doença ${ }^{(2)}$.

A baixa prevalência de percepção da discriminação nos serviços de saúde encontrada no atual estudo pode ser explicada pelo fato de que negros que compõem as comunidades tradicionais costumam procurar as religiões de matriz africana para os primeiros atendimentos de saúde ${ }^{(23,24)}$, reduzindo seu contato com os profissionais nas unidades de saúde, diminuindo a possibilidade de ocorrência do ato de discriminação(22).

A população negra brasileira residente nas áreas urbanas pode representar as maiores vítimas da discriminação por raça/cor praticada por profissionais de saúde. Nos espaços urbanos, os negros ocupam, majoritariamente, as periferias, locais com precariedade de bens e serviços, com exposição a situações de vulnerabilidade e que, frequentemente, são vinculadas a estigmas de violência, delinquência e desintegração. Essas características podem gerar uma postura preconceituosa, hostil e discriminatória por parte dos trabalhadores de saúde ante a figura de determinados grupos sociais negativamente estigmatizados, como é o caso da população negra ${ }^{(23-26)}$.

Ao se analisar a variável tabagismo, foi possível observar que pessoas que fumam sofreram mais discriminação quando comparadas àquelas que não fumam ou ex-fumantes. Logo, o estigma social atrelado ao tabaco e ao fumante, evidenciado pelo surgimento e consolidação das leis antitabagismo, gera marginalização dos usuários desse produto pelo maior conhecimento dos males causados por ele ou, ainda, pelo aumento da percepção da discriminação pelos próprios fumantes ${ }^{(27)}$.

O presente estudo também identificou maior proporção dos que relatam haver sofrido discriminação por raça/ cor entre os indivíduos que avaliam sua saúde como ruim/ muito ruim. A precariedade do serviço ofertado, em decorrência desse tipo de discriminação, pode ser fator determinante para o surgimento ou potencialização de agravos à saúde ou hábitos de vida inadequados $(7,9,11,12,28)$.

Evidências na literatura apontam que, mesmo sendo vítimas da discriminação por raça/cor, as pessoas com saúde mais frágil não desistem de procurar os serviços de saúde, pois sua situação torna a procura desses espaços indispensável, fazendo com que o evento estudado seja uma experiência particularmente prejudicial(12,29). Além disso, a discriminação por raça/cor por prestadores de cuidados de saúde pode contribuir para reduzir a satisfação do usuário em relação aos profissionais e ao serviço de saúde, o que pode provocar a não adesão aos tratamentos, repercutindo diretamente na efetividade do cuidado em saúde ${ }^{(12)}$.

Outro achado revela que utilizar o serviço público se relaciona à maior discriminação por raça/cor em serviços de saúde quando comparado às pessoas que possuem plano de saúde privado ${ }^{(10)}$. No Brasil, dados do Instituto de Pesquisa Econômica Aplicada apontam que, em 2008, $67 \%$ da população atendida no SUS era negra e que a faixa de renda de seus usuários estava entre um quarto e meio do salário mínimo. Considerando que a população negra constitui a maioria dos usuários do SUS e que a discriminação racial é mais prevalente entre usuários do serviço 
público, os achados desse estudo refletem uma preocupante fragilidade desse sistema, que reforça o racismo institucional já existente no panorama brasileiro ${ }^{(30)}$.

Desse modo, ressalta-se a relevância desses dados para efetivação de objetivos já pactuados pelo SUS através da Política Nacional de Promoção em Saúde, revisada em 2014, como a adoção de práticas sociais e de saúde centradas na equidade, participação e no controle social, buscando reduzir as desigualdades sistemáticas, injustas e evitáveis com respeito às diferenças, entre elas, as étnico-raciais. Portanto, as evidências do presente estudo permitem detectar uma ruptura dos princípios do sistema de saúde brasileiro e apontam para a necessidade de ampliar as discussões sobre o tratamento equitativo a grupos vulneráveis nesse espaço.

A discriminação por raça/cor, enquanto fenômeno subjetivo, pressupõe difícil aferição e captação, de modo que é um tema complexo a ser debatido em uma pesquisa quantitativa. Além disso, o viés de memória, o constrangimento provocado pelo questionamento e o extenso questionário da PNS podem reduzir ou aumentar o número de respostas positivas ou negativas a essa questão, configurando-se como limitações do presente estudo.

Por outro lado, o número restrito de pesquisas com desenho quantitativo e de associação na perspectiva da discriminação de raça/cor em serviços de saúde, no território brasileiro, reforça a relevante contribuição destes resultados para a ampliação do debate.

\section{CONCLUSÃO}

Foi possível identificar que a discriminação por raça/cor praticada por prestadores de cuidados de saúde no Brasil apresenta baixa prevalência, o que pode estar relacionado às construções e sanções culturais, sociais e legais envolvidas nesse fenômeno.

O evento estudado acontece de forma difusa em todas as regiões do Brasil, atinge especialmente a população negra, independente do sexo, e pessoas com vulnerabilidade em sua condição de saúde, com múltiplas morbidades, tabagistas, com autoavaliação de saúde ruim/ muito ruim e que utilizam exclusivamente o SUS.

\section{CONFLITOS DE INTERESSE}

Os autores declararam não haver conflitos de interesses.

\section{CONTRIBUIÇÕES}

Marianny Nayara Paiva Dantas e Isabelle Ribeiro Barbosa contribuíram com a elaboração e delineamento do estudo; a aquisição, análise e interpretação de dados; e a redação e/ou revisão do manuscrito. Kezauyn Miranda Aiquoc, Emelynne Gabrielly de Oliveira Santos e Nayre Beatriz Martiniano de Medeiros contribuíram com a aquisição, análise e interpretação de dados; e a redação e/ou revisão do manuscrito. Mercês de Fátima dos Santos Silva contribuiu com a aquisição, análise e interpretação dos dados. Dyego Leandro Bezerra de Souza contribuiu com a elaboração e delineamento do estudo e com a aquisição, análise e interpretação dos dados.

\section{REFERÊNCIAS}

1. Barata RB. Como e por que as desigualdades sociais fazem mal à saúde [Internet]. Rio de Janeiro: Editora FIOCRUZ; 2009 [acesso em 2019 Mar 18]. Disponível em: https://static.scielo.org/scielobooks/48z26/pdf/ barata-9788575413913.pdf

2. Paradies $Y$, Troung $M$, Prient $N$. A systematic review of the extent and measurement of healthcare provider racism. J Gen Intern Med [Internet]. 2013 [acesso em 2019 Mar 18];29(2):364-87. Disponível em: https://www. ncbi.nlm.nih.gov/pmc/articles/PMC3912280/

3. Werneck J. Racismo institucional e saúde da população negra. Saúde Soc [Internet]. 2016 [acesso em 2019 Mar 18];25(3):535-49. Disponível em: http://www.scielo.br/pdf/sausoc/v25n3/1984-0470-sausoc-25-03-00535. pdf

4. Almeida S. O que é racismo estrutural? Belo Horizonte: Letramento; 2018.

5. Instituto Brasileiro de Geografia e Estatística. Censo demográfico 2010: características da população e dos domicílios. Rio de Janeiro: IBGE; 2011.

6. Instituto Brasileiro de Geografia e Estatística. Pesquisa nacional por amostra de domicílios, 2015: síntese de indicadores. Rio de Janeiro: IBGE; 2016. 
7. Sellers SL, Cherepanov D, Hanmer J, Fryback DG, Palta M. Interpersonal discrimination and healthrelated quality of life among black and white men and women in the United States. Qual Life Res [Internet]. 2013 [acesso em 2019 Mar 18];22(6):1313-8. Disponível em: https://www.ncbi.nlm.nih.gov/pmc/articles/ PMC3978034/

8. Smith-Bynum MA, Lambert SF, English D, lalongo LS. Associations between trajectories of perceived racial discrimination and psychological symptoms among African American adolescents. Dev Psychopathol [Internet]. 2014 [acesso em 2019 Mar 18];26(4):1049-1065. Disponível em: https://www.ncbi.nlm.nih.gov/ pubmed/24955844

9. Kreling MCG, Pimenta CAM, Garanhani ML. Racial discrimination in pain management. Rev Dor [Internet]. 2015 [acesso em 2019 Mar 18];15(3):230-514. Disponível em: http://www.scielo.br/scielo.php?script=sci_artte xt\&pid=S1806-00132014000300230

10. Mays M, Jones AL, Delany-Brumsey A, Coles C, Cochran SD. Perceived Discrimination in Healthcare and Mental Health/ Substance Abuse Treatment Among Blacks, Latinos, and Whites. Med Care [Internet]. 2017 [acesso em 2019 Mar 18];55(2):173-81. Disponível em: https://www.ncbi.nlm.nih.gov/pubmed/27753743

11. Smolen JR, Araújo EM. Raça/cor da pele e transtornos mentais no Brasil: uma revisão sistemática. Ciênc Saúde Colet [Internet]. 2017 [acesso em 2019 Mar 25];22(12):4021-30. Disponível em: http://www.scielo.br/ scielo.php?script=sci_abstract\&pid=S1413-81232017021204021\&lng=pt\&nrm=iso

12. Ben J, Cormack D, Harris R, Paradies Y. Racism and health service utilisation: a systematic review and metaanalysis. PLoS ONE [Internet]. 2017 [acesso em 2019 Mar 25];12(12). Disponível em: https://www.ncbi.nlm. nih.gov/pubmed/29253855

13. Brasil MS. Indicadores de Vigilância em Saúde descritos segundo a variável raça/ cor, Brasil. Bol Epidemiol [Internet]. 2017 [acesso em 2019 Mar 25];48(4):1-35. Disponível em: http://portalarquivos2.saude.gov.br/ images/pdf/2017/fevereiro/17/Indicadores-de-Vigilancia-em-Saude-descritos-segundo-ra--a-cor.pdf

14. Batista WM, Mastrodi J. Dos fundamentos extraeconômicos do racismo no Brasil. Rev Direito Prax [Internet]. 2018 [acesso em 2019 Abr 10];9(4):2332-59. Disponível em: http://www.scielo.br/pdf/rdp/v9n4/2179-8966rdp-9-4-2332.pdf

15. Ministério Público do Trabalho do Estado do Paraná. MPT reforça promoção da inclusão de pessoas negras no mercado de trabalho [Internet]. 2019 [acesso em 2019 Nov 06]. Disponível em: http://www.prt9.mpt.mp.br/ informe-se/noticias-do-mpt-pr/45-noticias-prt-curitiba/1187-mpt-reforca-promocao-da-inclusao-de-pessoasnegras-no-mercado-de-trabalho

16. Campos LA. Racismo em três dimensões: uma abordagem realista-crítica. Rev Bras Ciênc Soc [Internet]. 2017 [acesso em 2019 Nov 06];32(95):329507. Disponível em: http://www.scielo.br/scielo.php?script=sci_ abstract\&pid=S0102-69092017000300503\&lng=pt\&nrm=iso\&tlng=pt

17. Souza-Júnior PRB, Freitas MPS, Antonaci GA, Szwarcwald CL. Desenho da amostra da Pesquisa Nacional de Saúde 2013. Epidemiol Serv Saúde [Internet]. 2015 [acesso em 2019 Nov 06];24(2):207-16. Disponível em: http://www.scielo.br/scielo.php?pid=S2237-96222015000200207\&script=sci_abstract\&tlng=pt

18. Instituto Brasileiro de Geografia e Estatística. Pesquisa Nacional de Saúde, 2013: questionário dos moradores do domicílio. Rio de Janeiro: IBGE; 2014.

19. Boutwell BB, Nedelec JL, Winegard B, Shackelford T, Beaver KM, Vaughn M, et al. The prevalence of discrimination across racial groups in contemporary America: Results from a nationally representative sample of adults. PLoS ONE [Internet]. 2017 [acesso em 2019 Nov 06];12(8). Disponível em: https://journals.plos.org/ plosone/article?id=10.1371/journal. pone. 0183356

20. Abramson CM, Hashemi M, Sánchez-Jankowski M. Perceived discrimination in U.S. healthcare: Charting the effects of key social characteristics within and across racial groups. Prev Med Rep [Internet]. 2015 [acesso em 2019 Nov 06];2:615-21. Disponível em: https://www.ncbi.nlm.nih.gov/pmc/articles/PMC4721495/

21. Rosa AR. Relações raciais e estudos organizacionais no Brasil. Rev Adm Contemp [Internet]. 2014 [acesso em 2019 Nov 06];18(3):241-259. Disponível em: http://www.scielo.br/pdf/rac/v18n3/v18n3a02.pdf

22. Tavares NO, Oliveira LV, Lages SRC. A Percepção de Psicólogos sobre o Racismo Institucional em Saúde Pública. Saúde Debate [Internet]. 2013 [acesso em 2019 Nov 06];37(99):580-7. Disponível em: http://www. 
scielo.br/scielo.php?pid=S0103-11042013000400005\&script=sci_abstract\&tlng=pt

23. Alves MC, Semitontine N. Health Care in a Community of Followers of Traditional African-Brazilian Religions. Rev Saúde Pública [Internet]. 2009 [acesso em 2019 Nov 06];43(Supl1):85-91. Disponível em: https://www. ncbi.nlm.nih.gov/pubmed/19669069

24. Osypuk TL, Garcia AD. Beyond Individual Neighborhoods: a Geography of Opportunity Perspective for Understanding Racial/Ethnic Health Disparities. Health Place [Internet]. 2010 [acesso em 2019 Nov 06];16(6):1113-23. Disponível em: https://www.ncbi.nlm.nih.gov/pmc/articles/PMC2952656/

25. Abreu IS. Biopolítica e racismo ambiental no Brasil: a exclusão ambiental dos cidadãos. Opin Jurid [Internet]. 2013 [acesso em 2019 Nov 06];12(24):87-99. Disponível em: http://www.scielo.org.co/scielo.php?script=sci_ abstract\&pid=S1692-25302013000200006\&lng=e\&nrm=iso\&tlng=e

26. Texeira S, Zuberi A. Mapping the Racial Inequality in Place: using youth perceptions to identify unequal exposure to neighborhood environmental hazards. Int J Environ Res Public Health [Internet]. 2016 [acesso em 2019 Nov 06];13(9):844. Disponível em: https://www.ncbi.nlm.nih.gov/pmc/articles/PMC5036677/

27. Lundberg GJW, Neel R, Lassetter B, Todd AR. Raciais bias in implicit danger associations generalizes to older male targets. PLoS ONE [Internet]. 2018 [acesso em 2019 Nov 06];13(6). Disponível em: https://www. ncbi.nlm.nih.gov/pubmed/29874255

28. Spink MJP. Ser fumante em um mundo antitabaco: reflexões sobre riscos e exclusão social. Saude Soc [Internet]. 2010 [acesso em 2019 Nov 06];19(3):481-49. Disponível em: http://www.scielo.br/scielo. php?script=sci_arttext\&pid=S0104-12902010000300002

29. Cuevas AG, Reitzel LR, Cao Y, Nguyen N, Wetter DW, Adams CE, et al. Mediators of Discrimination and Self-rated Health among African Americans. Am J Health Behav [Internet]. 2013 [acesso em 2019 Nov 06];37(6):745-5. Disponível em: https://www.ncbi.nlm.nih.gov/pubmed/24001623

30. Fazeli Dehkordy S, Hall KS, Dalton VK, Carlos RC. The link between everyday discrimination, healthcare utilization, and health status among a national sample of women. J womens Health [Internet]. 2016 [acesso em 2019 Nov 06];25(10):1044-51. Disponível em: https://www.ncbi.nlm.nih.gov/pubmed/27429363

31. Instituto de Pesquisa Econômica Aplicada. Retrato das desigualdades de gênero e raça [Internet]. $4^{a}$ ed. Brasília: Ipea; 2011 [acesso em 2019 Mar 18]. Disponível em: http://www.ipea.gov.br/portal/index. php?option=com_content\&view=article\&id $=12893$

32. Conselho Nacional de Secretários de Saúde (BR). Revisão da política nacional de promoção da saúde [Internet]. Brasília: CONASS; 2014 [acesso em 2019 Mar 18]. Disponível em: https://www.conass.org.br/wpcontent/uploads/2014/11/NT-18-2014-Politica-de-Promoc\%CC\%A7a\%CC\%83o-da-Sau\%CC\%81de.pdf

\author{
Endereço do primeiro autor: \\ Marianny Nayara Paiva Dantas \\ Universidade Federal do Rio Grande do Norte - UFRN \\ Programa de Pós-graduação em Saúde Coletiva \\ Avenida Senador Salgado Filho, 1787 \\ Bairro: Lagoa Nova \\ CEP: 59010-000 - Natal - RN - Brasil \\ E-mail: mariannynayara@hotmail.com

\section{Endereço para correspondência:} \\ Nayre Beatriz Martiniano de Medeiros \\ Universidade Federal do Rio Grande do Norte - UFRN \\ Programa de Pós-graduação em Saúde Coletiva \\ Avenida Senador Salgado Filho, 1787 \\ Bairro: Lagoa Nova \\ CEP: 59010-000 - Natal - RN - Brasil \\ E-mail: nayremartiniano@gmail.com
}

Como citar: Dantas MNP, Aiquoc KM, Santos EGO, Silva MFS, Souza DLB, Medeiros NBM, et al. Prevalência e fatores associados à discriminação racial percebida nos serviços de saúde do Brasil. Rev Bras Promoç Saúde. 2019;32:9764. 